\title{
Simplifying the Assessment of Building Vulnerability To Chemical, Biological, and Radiological Releases
}

\author{
Tracy L. Thatcher, Emily E. Wood, \\ Eve C. Edelson and Richard G. Sextro \\ Environmental Energy Technologies Division \\ Indoor Environment Department \\ Lawrence Berkeley National Laboratory \\ Berkeley, CA 94720
}

January 2005

This work was supported by the California Energy Commission's Public Interest Energy Research (PIER) program and performed under U.S. Department of Energy Contract No. DE-AC03-76SF00098. 


\title{
Simplifying the Assessment of Building Vulnerability To Chemical, Biological, and Radiological Releases
}

\author{
by Tracy L. Thatcher, Emily E. Wood, Eve C. Edelson and Richard G. Sextro
}

The intentional or accidental release of airborne chemical, biological, or radiological materials can pose a significant threat to the health of building occupants. Pre-planning and emergency response measures, such as HVAC system manipulation and sheltering during an event, can significantly reduce the exposure of building occupants. A straightforward and comprehensive vulnerability assessment methodology is an essential tool for assisting building managers and operators in preparing for airborne hazards.

In a world filled with conflicting demands for increased occupant comfort, building safety, operating efficiency, and cost reduction, a building manager can feel pushed in several directions at once. New mandates for the protection of buildings against chemical, biological, and radiological (CBR) agents make the situation even more complex, especially since this is an unfamiliar area for many building managers. While building managers are typically experts at providing services for their occupants, such as Heating, Ventilation, and Air Conditioning system (HVAC), security, and alarm systems, using their building expertise to reduce the threat from chemical, biological, and radiological materials is generally a new concept.

In the case of airborne CBR agents, the Heating, Ventilation, and Air Conditioning system (HVAC) is often the first line of defense. Preplanning and HVAC manipulation can significantly reduce the severity of a chemical, biological, or radiological attack or an accidental release of toxic materials. Several organizations have developed guidance for reducing a building's vulnerability to CBR agents ${ }^{1,2,3,4}$. These documents detail changes that can be made to building operations, HVAC systems, security, and response planning to prepare buildings to face the challenges posed by CBR threats.

The available guidance, while a valuable resource, can be difficult to apply. For instance, if a facility manager determines that there are a number of actions that could be taken to improve the preparedness of the building, the guidance doesn't help determine which ones to do first. Choosing the best course of action depends on both the cost of the improvement and the type and level of threat a facility faces. Due to the complexity of the issue, facility operators sometimes feel compelled to hire consultants to perform a site specific analysis to determine building specific recommendations, even for relatively low risk sites.

\section{Self-Assessment for CBR Vulnerability}

To make it easier for facility managers to respond to today's security needs, an interactive program for assessing a building's vulnerability to CBR attack was needed. The program's goal was to provide a user friendly, efficient, and field-tested program that could be used not only to assess vulnerability, but also to provide specific recommendations for a given building while 
minimizing the energy penalties sometimes associated with improving HVAC system security. The result was the Building Vulnerability Assessment and Mitigation Program (BVAMP), a program that provides state-of-the-art advice in an easy to use format. BVAMP can be obtained free through a website providing advice on CBR threats to buildings (http://securebuildings.lbl.gov). Details regarding the development and design of BVAMP are given in a freely available project report ${ }^{5}$.

Determining the vulnerability for a specific building requires that information about the building's operations and procedures be gathered. BVAMP provides a worksheet is provided to help with the information gathering phase, although many of the questions can be answered based on the knowledge facility managers will already have. The questions focus on areas critical to protection against chemical, biological, or radiological agents and are divided into five major topic areas: risk assessment, emergency response plan, building access, HVAC systems and HVAC controls. BVAMP is set up using a hierarchical approach based on yes/no answers. If the first level questions are answered "yes," a series of follow-up questions are pursued; if the answer is "no," then the program goes on to the next series of questions.

Once the questionnaire is completed, BVAMP produces a report that details any areas where the owner could increase the resistance of their building to CBR agents and prioritizes these recommendations both in terms of relative cost (high vs. low) and the threat level that would warrant implementation. Mangers can use the report recommendations to determine what actions, if any, are appropriate for their facility. In some cases, the recommended actions may require additional analysis or engineering studies to detail the nature and cost of the building preparedness actions. These are beyond the scope of the more general approach and recommendations contained in BVAMP.

\section{Know your Building}

By knowing their building and preparing ahead of time, building managers can reduce the likelihood and severity of a potential chemical, biological, or radiological attack. Steps to reduce the vulnerability of a facility to CBR agents fall into three general categories: HVAC system control and operation, building system security, and emergency response planning.

The importance of the first of these groups, HVAC system control and operation, is often overlooked when preparing for physical security threats, such as explosions or intruders. One of the most frequently considered methods for utilizing the HVAC system to reduce vulnerability to CBR agents is through improved filtration. There are two main classes of filters, particle filters and chemical (gas) filters. Of these, the first is far more commonly employed in buildings than the second. Particle filters vary in both type and particle removal efficiency. In most commercial buildings, filters with moderate removal efficiency (50\% dust spot or MERV 10) are typical. In some cases, upgraded filter efficiency can be achieved simply by installing higher efficiency filters, without requiring additional HVAC system modifications. Depending on the type of filters currently used and the type of higher efficiency filters chosen, upgrading filter efficiency may produce a higher pressure drop than the system can accommodate, thus reducing airflow and requiring additional air handler capacity. This problem can often be reduced or eliminated by choosing a deeper filter, such as a pleated filter. It is important to remember that 
particle filters only protect occupants from particulate contaminates (such as biological materials) and will not reduce exposures to toxic gases.

The technology that removes the widest range of toxic gases from airstreams uses treated activated carbon filters. However, these chemical filtration systems typically impose a significant pressure drop on an HVAC system and are not widely used. They also have fairly high replacement costs and are not effective against all types of chemicals. If protection against chemicals is needed, an alternative to filtration of the entire building is establishing a safe room or shelter area that limits air exchange with the out-of-doors and installing chemical filters for just that area. This may be of particular interest in buildings that are near a facility that has a high risk of either accidental or intentional release (such as a major freight line, industrial facility, or high threat building).

\section{Determining Risk}

Determining risk or threat for a specific building is a complex matter. The terrorist threat risk is influenced by the nature of the activities performed in the building (whether there are terrorists who would consider that facility a particularly good target), the number of occupants (increased occupancy tends to lead to increased risk), and historical or other special building status (wellknown or symbolic buildings may be at increased risk). In buildings that use or store significant quantities of chemical, radiological and/or biological materials, occupants are at increased risk of injury or death from an accidental release and may require added precautions to protect against indoor releases.

The threat level for a facility is also influenced by the buildings and activities in the surrounding area. Being near a facility with a high terrorist risk or large quantities of CBR materials puts a building at an increased risk of being impacted by an attack or accident at these nearby facilities. Buildings near high-risk facilities need to take added precautions to reduce the impact of external contaminants. For example, a building near a chemical processing facility may decide to go to the added expense of providing shelter-in-place areas with chemical filtration units capable of removing the most hazardous materials used at the processing facility. However, a building that is at low risk from external contaminant plumes may prefer to use safety funds for other measures that are identified as being more probable in their situation.

Local law enforcement and emergency response planning agencies can assist facility managers with determining the terrorist risk level for a facility and determining the threat posed by other local facilities.

\section{Outdoor CBR Release}

During an accidental or intentional outdoor CBR release, it may not be possible to safely evacuate building occupants. In most cases, occupants will need to stay indoors until the chemical plume passes the building. While the building itself will provide some protection, modifying the operation of the HVAC system during an emergency can significantly reduce the impact of a CBR release, potentially saving lives and reducing property contamination. For example, if a tank car carrying a toxic chemical overturns near a facility, turning off the HVAC 
system will reduce the concentration indoors. Figure 1 illustrates the potential effectiveness of sheltering within a building during an outdoor event. Since quick and efficient evacuation of densely populated areas is typically not possible, occupants are more likely to survive if they shelter in a building which has prepared for CBR emergencies and where the HVAC system can be quickly shutdown to reduce indoor exposures.

Reducing the air exchange rate, or leakiness, of a building can further reduce the exposure of occupants to an accidental or intentional outdoor release of chemical, biological or radiological materials. Lowering air exchange rates reduces the speed at which contaminants enter a building, thereby lowering indoor concentration and increasing the length of time that occupants can safely shelter indoors. Reducing building leakage may also improve comfort (through elimination of drafts), improve moisture control, and increase energy efficiency - depending on current building conditions. Reducing the airflow between high-risk areas, such as lobbies and mailrooms, and the rest of the building can reduce the transport of an indoor release in these zones to occupants in other areas.

\section{Indoor CBR Release}

In the event of a chemical release indoors, a different HVAC system strategy should be used. Increasing the outside air supply and eliminating recirculation of return air into the building supply air reduces both the spread of the chemical via the HVAC system and the concentration near the chemical source. Figure 2 shows how a hazardous agent release in one area (in this example the first floor) can be transported quickly throughout the entire zone, if there is a common return and the return air is recirculated. For maximum impact, it is important that HVAC operations can be manipulated quickly and easily during an emergency. Ideally, both sheltering and purging HVAC system responses will be pre-programmed for quick and easy implementation during an emergency.

\section{Reducing Vulnerability}

Since HVAC system manipulation is crucial for minimizing the impact of a CBR attack, the integrity of the HVAC system must be maintained. This means that additional precautions need to be taken to assure that only authorized personnel have access to the HVAC system, system controls, and plans. Additionally, emergency response teams must include personnel who understand and can manipulate HVAC fans and dampers.

In addition to improved HVAC control security, increasing the security of building systems and building system information is also desirable. By making it more difficult for potential terrorists to gain access to, and find information about, your building, you increase the likelihood that they will be caught while planning an attack. Many of the actions needed to improve security represent very low costs. However, they may require changes in the way building management shares information and therefore be difficult to implement. The recommendations in BVAMP deal with security as it pertains to HVAC and CBR agent vulnerabilities. They do not deal with threats posed by bombs, thefts, or other issues, which can be addressed through other programs and by local law enforcement agencies. 
The third key aspect of vulnerability reduction and mitigation for buildings is emergency response planning. Up-to-date and complete emergency response plans are a crucial component of any vulnerability reduction strategy. Pre-planning and employee awareness, coupled with a well thought out and rehearsed emergency plan can reduce confusion and save lives during an actual emergency. Many of the planning measures recommended for preparing for CBR attack are also helpful for responding to other types of emergencies, such as fires or tornadoes.

Although emergency response planning for CBR releases is similar to that for other emergency situations, there are several significant differences. For example, emergency communication is more critical. Since indoor releases require different responses than outdoor releases, communication with occupants needs to be able to convey more information than a simple fire alarm bell can provide. In addition, it is important to have a choice of evacuation locations, depending on the wind direction. Otherwise, occupants evacuating the building could be exposed to the agent as it exhausts from the building. Building occupants should understand that evacuation routes and staging areas may differ, depending on the specific emergency, and emergency drills should include both evacuating and sheltering in place. In addition, plans should be made to assist employees with special needs during both evacuation and sheltering. In general, it is acceptable to incorporate elevator use for disabled building occupants in CBR emergency evacuation plans, when there has been no physical damage to the building and this is the fastest evacuation mode for them.

\section{Vulnerability Assessment Report}

For building managers who want an easy-to-use, straightforward method for conducting a comprehensive vulnerability assessment, BVAMP is a useful tool. After answering the BVAMP questions, the program can generate a report describing potential areas of vulnerability and suggesting ways to address these vulnerabilities. The recommendations are divided into six sections: emergency response plans, shelter-in-place, HVAC systems, air exchange rate reduction, security, and special risk areas. For each section, specific recommendations are separated into groups based on both the probable cost of implementation (high or low) and the type and level of risk that would warrant implementation.

\section{Addressing Vulnerabilities Can Save Lives}

Although the probability of a chemical, biological, or radiological event in or near a building is low, the consequences of such an event could be catastrophic. Accidents involving tanker cars, trucks carrying hazardous materials, chemical manufacturing facilities, and refineries are among the historical risks many facilities face. Now the risk of deliberate attack by terrorists wielding hazardous materials presents additional concerns for buildings and their occupants. Fortunately, there are numerous steps that facility managers can take to reduce these risks. For many facilities, significant vulnerability reductions can be obtained through implementation of low cost mitigations, such as improvements in emergency response plans.

The Building Vulnerability Assessment and Mitigation Program (BVAMP) is the first fieldtested, easy-to-use protocol that building owners and operators can utilize to reduce the likelihood and severity of a chemical, biological, or radiological event. BVAMP produces a building-specific vulnerability assessment report that explicitly considers the costs and energy 
impacts of mitigation measures and whether the measure is suggested for all buildings or is more appropriate for those buildings at increased risk. BVAMP can help building managers improve emergency preparedness in a cost effective and efficient manner. Increased preparedness will, in turn, provide positive benefits for reducing both the likelihood and the severity of chemical, biological, or radiological events.

\section{Acknowledgement}

This work was supported by the California Energy Commission's Public Interest Energy Research (PIER) program and performed under U.S. Department of Energy Contract No. DEAC03-76SF00098. 


\section{References}

1. American Society of Heating, Refrigerating, and Air-Conditioning Engineers (ASHRAE), Risk Management Guidance for Health and Safety under Extraordinary Incidents, January 2002.

2. Centers for Disease Control and Prevention, National Institute for Occupational Safety and Health (CDC/NIOSH) Guidance for Protecting Building Environments from Airborne Chemical, Biological, or Radiological Agents, DHHS(NIOSH) Publication No. 2002-139, May 2002.

3. U.S. Army Corps of Engineers, Protecting Buildings and their Occupants from Airborne Hazards, DRAFT TI 853-01, http://buildingprotection.sbccom.army.mil/basic/index.htm, October 2001.

4. U.S. Army Soldier and Biological Chemical Command (SBCCOM), Basic Information on Building Protection: Strategies for Protecting Buildings from Airborne Hazards, http://buildingprotection.sbccom.army.mil/basic/spbah.htm, November 2001.

5. Thatcher, T. L. and Sextro, R.G., Building Vulnerability Assessment and Mitigation Final Report, LBNL/PUB 56731 December 2004. 